\title{
3-Hydroxy-3-Methylglutaryl Coenzyme A Reductase Inhibitors Protect Cortical Neurons from Excitotoxicity
}

\author{
Anna Zacco, ${ }^{1}$ James Togo, ${ }^{1}$ Katherine Spence, ${ }^{1}$ Amanda Ellis, ${ }^{1}$ Darlene Lloyd, ${ }^{2}$ Steve Furlong, ${ }^{2}$ and Timothy Piser ${ }^{1}$ \\ Departments of ${ }^{1}$ Neuroscience and ${ }^{2}$ Molecular Sciences, CNS Discovery Research, AstraZeneca Pharmaceuticals, Wilmington, Delaware 19850
}

3-Hydroxy-3-methylglutaryl-CoA (HMG-CoA) reductase inhibitors, or statins, reduce the incidence of strokes and reduce infarct volume after cerebral ischemia in mice. Excitoxicity caused by overstimulation of glutamate receptors is a major cause of neuronal death after an ischemic brain insult. Experiments presented here explored whether statins protect cultured neurons from excitotoxic death caused by the glutamate receptor agonist NMDA. Treatment with statins preserved NMDA receptor-expressing cortical neurons and potently and substantially reduced lactate dehydrogenase release caused by exposure of embryonic mouse neocortical cultures to NMDA. The rank order of neuroprotective potency was rosuvastatin $=\operatorname{simvastatin}>$ atorvastatin $=$ mevastatin $>$ pravastatin, which is similar to the known rank order of potency for inhibition of the HMG-CoA reductase enzyme. Resistance of cultures to NMDA excitotoxicity developed after several days of statin exposure. Neuroprotection by rosuvastatin was coincident with a decrease in cell sterols and occurred with a similar potency as inhibition of cholesterol biosynthesis. Neuroprotection was substantially attenuated by cotreatment with either mevalonate or cholesterol and was mimicked by acute treatment with the cholesterol-extracting agent $\beta$-cyclodextrin, suggesting that neuroprotection was mediated by depletion of a cellular pool of cholesterol because of the inhibition of cholesterol biosynthesis. These results suggest the possibility that, in addition to effects on cerebrovascular function, statins have the potential to render cortical neurons more resistant to NMDA-induced excitotoxic death as a result of changes in cell cholesterol homeostasis.

Key words: HMG-CoA reductase; statin; cholesterol; NMDA; excitotoxicity; neuron

\section{Introduction}

3-Hydroxy-3-methylglutaryl-CoA (HMG-CoA) reductase inhibitors (statins) are potent cholesterol-lowering drugs. Several clinical studies indicate that statins reduce the incidence of stroke in some patients (Scandinavian Simvastatin Survival Study Group, 1994; Shepherd et al., 1995; Sacks et al., 1996; The LongTerm Intervention with Pravastatin in Ischemic Disease (LIPID) Study Group, 1998; Warshafsky et al., 1999; Heart Protection Study Collaborative Group, 2002). Retrospective case-control studies further suggest that the prevalence of Alzheimer's disease and vascular dementia is reduced among people taking statins (Jick et al., 2000; Wolozin et al., 2000). One interpretation of these findings is that statins may exert a broad neuroprotective effect.

Pretreatment with statins reduces infarct volume and provides protection from neurological deficits in mouse models of cerebral ischemia (Endres et al., 1998; Laufs et al., 2000, 2002; Amin-Hanjani et al., 2001). Studies in these models indicate that statins provide protection from ischemic brain damage by cerebrovascular mechanisms such as increased expression and activity of endothelial nitric oxide synthase (NOS) in endothelial cells and increased cerebral blood flow (Endres et al., 1998). In addi-

\footnotetext{
Received April 11, 2003; revised Sept. 26, 2003; accepted 0ct. 8, 2003.

We thank Charlotte Lott and My Linh Do for culture preparation and Drs. Fergus McTaggart, David Tuffin, and Adam Dudley for critical reading of this manuscript.

Correspondence should be addressed to Dr. Timothy Piser, Department of Neuroscience, AstraZeneca Pharmaceuticals, 1800 Concord Pike, Wilmington, DE 19850. E-mail: Timothy.Piser@AstraZeneca.com.

Copyright $\odot 2003$ Society for Neuroscience $\quad 0270-6474 / 03 / 2311104-08 \$ 15.00 / 0$
}

tion, several clinical studies have documented improved endothelial function during statin therapy, and human arterial blood flow is improved even in response to a single high dose of atorvastatin (Laufs et al., 2001). Thus, cerebrovascular mechanisms of action probably account, in part, for the reduced incidence of stroke in patients taking statins.

The current study describes another possible mechanism by which statins might directly protect neurons from ischemiainduced neurodegeneration by reducing neuronal cholesterol. Two recent clinical studies reported that some statins alter CSF markers of brain cholesterol homeostasis. For example, treatment with a high dose of simvastatin reduced the CSF level of the cholesterol biosynthetic intermediate lathosterol and the brain cholesterol-efflux metabolite 24-OH-cholesterol (Simons et al., 2002), and hypercholesterolemic patients treated with statins exhibited significantly lower CSF concentrations of both cholesterol and lathosterol (Fassbender et al., 2002). If these changes correspond to a decrease in neuronal membrane cholesterol, then some statins may alter membrane-delimited processes that mediate ischemic neuronal death such as NMDA receptor (NMDAR)-mediated excitotoxicity.

Excitotoxicity mediated by glutamate-gated ion channels is a well documented form of neuronal death caused by brain ischemia (Sattler and Tymianski, 2001). The NMDAR subtype of glutamate receptors probably plays the major role because of its high calcium permeability. Reducing neuronal membrane cholesterol could have profound effects on NMDAR-mediated processes. For example, NMDA excitotoxicity and ischemic neuro- 
degeneration is mediated, in part, by a tri-partite protein complex (Aarts et al., 2002) that resides in sterol-rich neuronal membrane microdomains known as lipid rafts (Perez and Bredt, 1998). Reducing cholesterol in cultured cells has been shown to alter the function and composition of several lipid-raftassociated neuronal protein complexes (Martens et al., 2000; Ehehalt et al., 2003). Furthermore, treatment with statins alters the function of lipid-raft-associated protein complexes in cultured neurons (Simons et al., 1998). These observations lead to the hypothesis that reducing neuronal cholesterol by inhibiting brain HMG-CoA reductase protects neurons from NMDARmediated excitotoxicity. The results presented here describe experiments designed to test this hypothesis using cultured neurons.

\section{Materials and Methods}

Drugs. Simvastatin, atorvastatin, and pravastatin were isolated from commercially available tablets using standard chemical extraction and purification techniques; simvastatin sodium salt was prepared by alkaline hydrolysis of the lactone form. The purity of active ingredient was determined to be: simvastatin lactone $(97.4 \%)$; atorvastatin $(81.4 \%)$; pravastatin $(87.1 \%)$. All analytical data were consistent with the known structures of these drugs, and in no case were any significant organic impurities detected, the remainder of the weight being water, solvent, and minor inorganic impurities. The neuroprotective potencies of simvastatin lactone and simvastatin sodium salt were identical (data not shown). Rosuvastatin calcium salt was synthesized at AstraZeneca Pharmaceuticals (Wilmington, DE). Mevastatin (compactin), mevalonate, cholesterol, NMDA, and $\beta$-cyclodextrin were obtained from Sigma (St. Louis, MO). Drug stocks were prepared in DMSO at 1000 -fold greater than experimental concentrations, unless indicated otherwise.

Cortical culture. Fetal mouse neocortical cells were cultured on a glial feeder layer. Cortices were harvested from E16 Swiss-Webster mouse embryos in ice-cold dissecting media [HBSS plus (in mM): 28 glucose, 10 sucrose, and 4.2 sodium bicarbonate]. To dissociate cortices into single cells, they were minced in $1 \mathrm{ml}$ of dissecting media, incubated in $0.09 \%$ trypsin in media stock (MS) [Eagle's MEM with Earle's salts plus (in mM): 22 glucose and 29 sodium bicarbonate] for $30 \mathrm{~min}$ at $37^{\circ} \mathrm{C}$, centrifuged at 2,500 rpm for $5 \mathrm{~min}$, resuspended in $1 \mathrm{ml}$ of MS, 5\% FBS, 5\% horse serum (HS), and triturated with glass pipettes. To prepare a glial feeder layer, cell suspensions similarly prepared from $2 \mathrm{~d}$ postnatal mouse cortices were plated at 0.5 cortices/plate in 24 -well tissue-culture plates that had been coated overnight with poly-D-lysine and laminin. These cultures produced an astrocyte monolayer on which cells prepared from E16 cortices were plated 2 weeks later at a density of 350,000 cells/well. After $6 \mathrm{~d}$ in culture, plating media were replaced with growth media containing $10 \% \mathrm{HS}$ and $10 \mu \mathrm{m}$ cytosine arabinoside (Ara-C); $2 \mathrm{~d}$ later, the culture media were again exchanged with fresh growth media lacking Ara-C. Typically, statins were added to the culture by media exchange on days 9 and 12. In some experiments, mevalonate (1 mM in PBS) and cholesterol (10 $\mathrm{mm}$ in ethanol) were added on the same days.

NMDA-induced lactate dehydrogenase release. Neuronal death was elicited $14-15 \mathrm{~d}$ after plating of embryonic cultures. Cultures were first washed twice with HEPES-buffered control salt solution (HCSS) (in mM: $120 \mathrm{NaCl}, 5.4 \mathrm{KCl}, 0.8 \mathrm{MgCl}_{2}, 1.8 \mathrm{CaCl}_{2}, 15$ glucose, 20 HEPES acid, 10 $\mathrm{NaOH}$, and $0.5 \%$ phenol red, $\mathrm{pH} 7.4$ ) to remove all drugs and then exposed to $300 \mu \mathrm{M}$ NMDA and $10 \mu \mathrm{M}$ glycine for $10 \mathrm{~min}$ in HCSS. Cultures were then washed three times and incubated overnight in MS. After $24 \mathrm{hr}$, a media sample was collected to determine lactate dehydrogenase $(\mathrm{LDH})$ activity. $\mathrm{LDH}$ activity was determined by measuring the linear rate of consumption of NADH absorption $(340 \mathrm{~nm})$ during the reduction of pyruvate to lactate using a spectrophotometer. Neuronal death was calculated from $\mathrm{LDH}$ activity by determining the percentage of the total NMDA-releasable pool that was measured for a given test condition using the following equation: neuronal death $=[($ A-Basal $) /($ MaxBasal) $] \times 100$, in which $A$ was the mean $\mathrm{LDH}$ activity measured in media samples from four wells subjected to the test condition, Max was the total

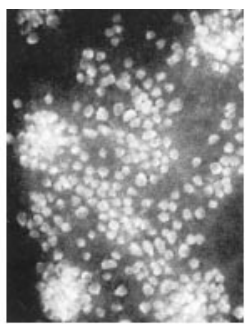

Control

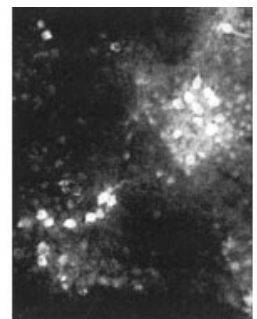

NMDA

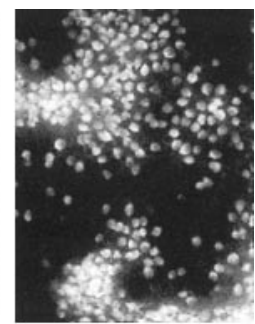

Simvastatin

+ NMDA

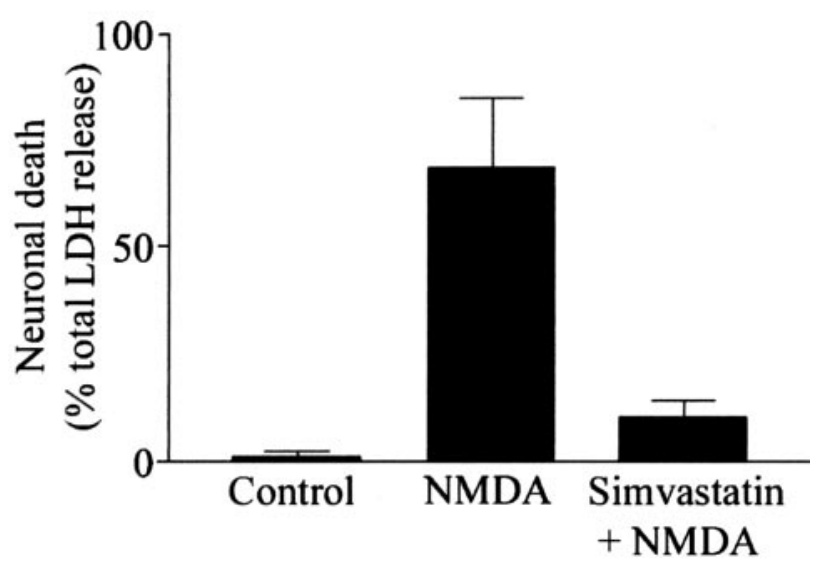

Figure 1. Simvastatin protects cultured mouse cortical neurons from NMDA excitotoxicity. Nine-day-old cortical cultures were treated with $100 \mathrm{~nm}$ simvastatin for $6 \mathrm{~d}$ or left untreated before a $10 \mathrm{~min}$ exposure to $300 \mu \mathrm{m}$ NMDA. $A$, fluorescence photomicrographs $(200 \mathrm{X})$ of NMDAR-1 immunocytochemically labeled cultures show that NMDAR-expressing neurons were preserved in cultures pretreated with simvastatin before NMDA exposure. $B, \mathrm{LDH}$ release, calculated as a percentage of the total NMDA-releasable pool of LDH, shows that NMDA-induced $\mathrm{LDH}$ release from the same cultures was prevented by simvastatin pretreatment.

NMDA-releasable pool of LDH activity observed in four wells treated for $24 \mathrm{hr}$ with $300 \mu \mathrm{M} \mathrm{NMDA}$, and Basal was the LDH activity from four identically treated wells that were not exposed to NMDA. These controls were run on every plate in each culture and averaged across multiple plates derived from the same culture. In all but Figure 1 (a representative single experiment), results are the mean of experiments conducted in three different cultures. In the concentration-response and time course experiments shown in Figure 2, consistent drug effects were observed, but the neuronal death caused by a 10 min exposure to NMDA varied somewhat between cultures. To calculate the effects of the drug independent of the variability in the effect of transient exposure to NMDA in the cultures used in these experiments, 10 min NMDA-induced neuronal death observed in drug-treated wells was normalized to 10 min NMDAinduced neuronal death observed in nondrug-treated wells in the same culture. These data are, therefore, reported as percentages of NMDAinduced neuronal death. In this system, NMDA-induced decreases in neuronal cell counts correlate with NMDA-induced increases in LDH release, and both are prevented by the noncompetitive NMDAR antagonist MK-801 (data not shown).

Immunocytochemistry. Cultures were washed with PBS for 5 min, fixed with $4 \%$ paraformaldehyde in PBS for 20 min, washed twice with PBS, incubated with ice-cold methanol for $10 \mathrm{~min}$, washed twice with PBS, then blocked with $10 \%$ donkey serum in PBS for $1-2 \mathrm{hr}$. Cultures were then incubated overnight in 1:50 rabbit NMDAR subtype 1 (NMDAR-1) antibody (Chemicon, Temecula, CA) in blocking solution at room temperature, washed three times with PBS, incubated for $1 \mathrm{hr}$ in 1:100 Cy3conjugated anti-rabbit IgG secondary antibody (Jackson Immunoresearch, West Grove, PA) in blocking solution at room temperature, and washed three times with PBS. Digital photomicrographs were obtained using an inverted Olympus (Tokyo, Japan) fluorescence microscope. 


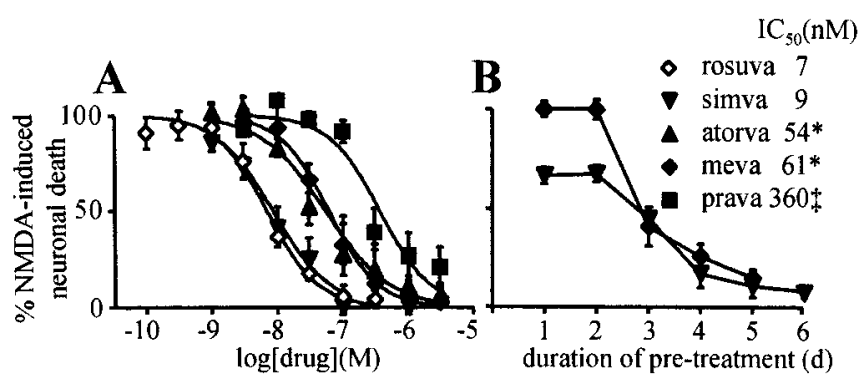

Figure 2. Statin-mediated neuroprotection is concentration and time dependent. $A$, Statins potently protect neurons from NMDA excitotoxicity, consistent with inhibition of HMG-COA reductase. Cortical cultures were treated with various concentrations of the indicated statins for $6 \mathrm{~d}$ before NMDA exposure. ${ }^{*}, p<0.05$ vs rosuvastatin; $\neq, p<0.01$ vs rosuvastatin. $B$, Statininduced neuroprotection developed over several days of pretreatment. Cortical cultures were exposed to either $100 \mathrm{~nm}$ simvastatin or $300 \mathrm{~nm}$ mevastatin for the indicated number of days before NMDA exposure.

Sterol Measurements. Cortical cultures treated with drugs as described above were saponified in ethanolic $\mathrm{KOH}$ and extracted with hexane. Extracts were derivatized to sterol esters using pyridine/acetic anhydride and analyzed by gas chromotography-mass spectroscopy. Cell culture sterols were identified and quantified by comparing retention times, spectra, and peak heights to those obtained with known amounts of authentic standards.

Electrophysiology. Both electrophysiology and calcium-imaging experiments were performed at room temperature on cultures grown on individual glass coverslips otherwise as described above. Before experiments, cultures were thoroughly washed free from drug-containing cell culture media using the relevant superfusion solutions. Currents were recorded using the whole-cell patch-clamp technique. Borosilicate glass pipettes with resistances of 5-7 M $\Omega$ were filled with the following solution (in mM): $135 \mathrm{CsCl}, 10$ EGTA, 10 HEPES, $1 \mathrm{MgCl}_{2}$, and $4 \mathrm{Mg}$-ATP, pH 7.4, with $\mathrm{CsOH}$ and adjusted to $305 \mathrm{mOsm}$ using sucrose. The superfusion solution contained the following (in $\mathrm{mM}$ ): $135 \mathrm{NaCl}, 5 \mathrm{KCl}, 2 \mathrm{CaCl}_{2}, 5$ HEPES, 11 glucose, and 0.01 glycine, $\mathrm{pH} 7.3$, with $\mathrm{NaOH}$ and adjusted to 315 mOsm using sucrose. Drugs were applied using a linear array of gravity-fed, glass-lined tubes connected to solenoid valves under digital control (BME Systems, Baltimore MD). Whole-cell capacitance $\left(C_{\mathrm{m}}=\right.$ integral of capacitive transient) was calculated by fitting currents recorded during a $-1 \mathrm{mV}, 10 \mathrm{msec}$ step. Membrane currents were amplified with an Axopatch 2A amplifier (Axon Instruments, Foster City, CA), low-pass filtered at $10 \mathrm{kHz}$, digitized at $2 \mathrm{kHz}$, and stored as computer files using P-Clamp software (Axon Instruments) and a TL-1 interface (Scientific Solutions, Solon, OH). Cell membrane potential was held at $-60 \mathrm{mV}$. NMDA $(100 \mu \mathrm{M})$ was applied for $1.6 \mathrm{sec}$ every $45 \mathrm{sec}$ after break-in. Peak current amplitudes were calculated by subtracting NMDA-evoked currents obtained in the presence of $100 \mu \mathrm{M} \mathrm{D}(-)-2-$ amino-5-phosphonopentanoic acid. A variable, brief, and modest rundown in peak NMDA current amplitude was observed. Reported peak amplitudes were derived from traces obtained after currents had stabilized.

Calcium Imaging. Calcium microfluorimetry experiments were conducted using Axon Imaging Workbench 2.2 and a Sensicam CCD camera (PCO CCD Imaging, Kelheim, Germany) attached to a Nikon Eclipse TE300 inverted fluorescence microscope. Cultures were incubated with the acetoxy-methyl-ester of the fluorescent calcium-indicator dye fura-2 (Molecular Probes, Eugene, OR) at $37^{\circ} \mathrm{C}$ for $45 \mathrm{~min}$, rinsed, then maintained in the dark at room temperature for an additional 15 min before use. Coverslips were attached with vacuum grease to a coverslip holder (Warner Instruments) and superfused for the remainder of the experiment with the following solution (in $\mathrm{mM}$ ): $135 \mathrm{NaCl}, 5 \mathrm{KCl}, 2 \mathrm{CaCl}_{2}, 5$ HEPES, and 11 glucose, $\mathrm{pH} 7.3$; osmolarity adjusted to 315 mosM with sucrose and $200 \mathrm{~nm}$ TTX. The excitation exposure interval was adjusted to 3-10 msec to obtain fluorescence that was dim but distinct during excitation at $340 \mathrm{~nm}$ and less than half-maximal during excitation at 380 $\mathrm{nm}$. Intraneuronal calcium concentration is proportional to the ratio (R) of the fura-2 fluorescence emission intensity measurements obtained when the dye is excited at 340 or $380 \mathrm{~nm}$ excitation wavelengths $\left(F_{340} /\right.$ $F_{380}$ ). Ratio images were collected at $5 \mathrm{sec}$ intervals to determine baseline fluorescence, then images were collected at $1 \mathrm{sec}$ intervals for $\sim 1 \mathrm{~min}$ during superfusion with $300 \mu \mathrm{M}$ NMDA and $10 \mu \mathrm{M}$ glycine. After the peak response was obtained, the sampling interval was increased to $5 \mathrm{sec}$. Baseline and peak values were determined for each cell from individual traces off-line. Cells that were unresponsive or exhibited either an unstable baseline or an oscillating response to NMDA were excluded from analysis. For statistical comparisons, the peak NMDA-induced increase in intraneuronal calcium concentration $(\Delta R)$ was measured in $\sim 20$ neurons per microscope field and was averaged to generate a single value for each coverslip.

Statistics and pharmacology. Concentration-response curves were fitted using a four-parameter, sigmoidal concentration-response equation of the following form: $y=$ Bottom $+[($ Top - Bottom $) /(1+$ $\left.\left.10^{\left(\log I C 50-[\mathrm{drug}]^{*} \mathrm{n}\right)}\right)\right]$. Statistical significance was determined using one of three tests. For electrophysiological and calcium-imaging studies, in which comparisons were made between mean values calculated for the two treatment groups, Student's $t$ test was performed. For all other experiments, in which the mean values of multiple treatment groups were compared, an ANOVA was conducted to determine whether significant differences existed between the means. If the ANOVA reached significance $(p<0.05)$, then either a Dunnett's test (all means compared with a control) or a Bonferonni test (comparisons between individual means) was used.

\section{Results}

Simvastatin preserves NMDAR-1 immunoreactivity and reduces $\mathrm{LDH}$ release from NMDA-treated cortical cultures

Dissociated embryonic mouse neocortices were plated on a 2-week-old glial feeder layer derived from neonatal mice. Nine days later, cultures were treated with $100 \mathrm{~nm}$ of the HMG-CoA reductase inhibitor simvastatin. After $6 \mathrm{~d}$ of exposure to simvastatin, cultures were washed and exposed to $300 \mu \mathrm{M}$ NMDA for $10 \mathrm{~min}$. To determine the survival of cortical neurons under these conditions, both NMDAR-1 immunofluorescence microscopy and LDH release measurements were conducted $24 \mathrm{hr}$ later in the identical simvastatin-treated and control cultures. As shown in Figure 1, $6 \mathrm{~d}$ of pretreatment with $100 \mathrm{~nm}$ simvastatin protected cortical neurons from NMDA-induced death, as indicated by the preservation of NMDAR-1 immunoreactive neurons, and a concomitant decrease in NMDA-induced $\mathrm{LDH}$ release in simvastatin-treated cultures.

In the representative experiment shown in Figure 1, exposure to $300 \mu \mathrm{M}$ NMDA for $10 \mathrm{~min}$ elicited release of $69 \pm 16 \%$ of maximal NMDA-induced LDH release, which occurred after a 24 hr exposure to $300 \mu \mathrm{M} \mathrm{NMDA}$, and is subsequently referred to as "total LDH." Basal LDH release was defined as that measured in cultures subjected to the identical wash protocol but without exposure to NMDA, which was $1.1 \pm 0.9 \%$ of the maximum. Pretreatment with $100 \mathrm{~nm}$ simvastatin, a maximally neuroprotective concentration of simvastatin, reduced NMDA-induced LDH release to $10 \pm 4 \%$ of total $\mathrm{LDH}(p<0.01)$. Simvastatin produced a modest, and nonsignificant, decrease in basal LDH release (data not shown).

\section{Concentration and time dependence of neuroprotection by statins}

Under identical experimental conditions, the neuroprotective concentration-response relationship was determined for five HMG-CoA reductase inhibitors. As shown in Figure 2, all five drugs exhibited concentration-dependent and potent inhibition of NMDA-induced excitotoxicity. The drugs produced equivalent and nearly complete maximal neuroprotection but differed 


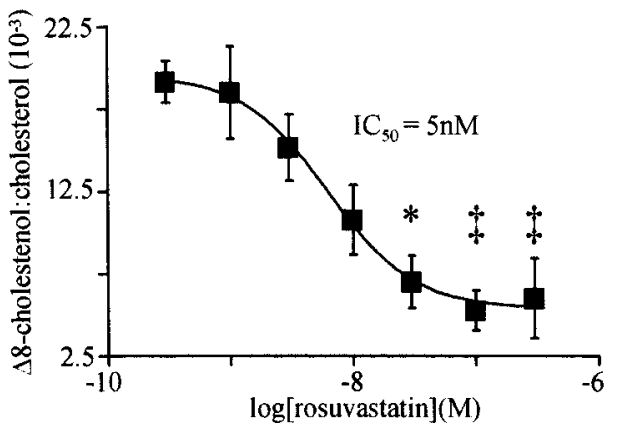

Figure 3. Rosuvastatin potently inhibits cortical culture cholesterol biosynthesis. $\Delta-8$ Cholestenol and cholesterol were measured in cortical cultures treated for $3 \mathrm{~d}$ with the indicated concentrations of rosuvastatin. ${ }^{*}, p<0.05 ; \ddagger, p<0.01$ vs vehicle-treated cultures.

in their neuroprotective potency. The rank order of neuroprotective potency was rosuvastatin $=$ simvastatin $>$ atorvastatin $=$ mevastatin $>$ pravastatin. The $\mathrm{IC}_{50}$ values and the $95 \%$ confidence intervals of the $\mathrm{IC}_{50}$ values for inhibition of NMDAinduced neuronal death were 7 (5-10), 9 (6-13), 54 (32-91), 61 (50-74), and $363(171-771) \mathrm{nm}$, for rosuvastatin, simvastatin, mevastatin, atorvastatin, and pravastatin, respectively (Fig. 2A). The potency of rosuvastatin and simvastatin was not statistically different, but mevastatin $(p<0.05)$, atorvastatin $(p<0.05)$, and pravastatin $(p<0.01)$ were less potent than rosuvastatin and simvastatin, and pravastatin was less potent than either atorvastatin or mevastatin $(p<0.05$ in both cases). The time course of neuroprotection was determined by treating cultures with either $100 \mathrm{~nm}$ simvastatin or $300 \mathrm{~nm}$ mevastatin for 1-6 d before NMDA exposure on day 15 in culture. As shown in Figure $2 B$, most of the neuroprotection afforded by either drug developed between 2 and $4 \mathrm{~d}$ of pretreatment.

\section{Cortical culture sterol analysis}

To determine whether statins protect neurons from excitotoxicity by inhibiting cholesterol biosynthesis, the effect of rosuvastatin treatment on cortical culture sterol levels was measured. Treatment with rosuvastatin reduced cortical culture cholesterol and $\Delta$-8-cholestenol and decreased the ratio of $\Delta$-8-cholestenol to cholesterol. $\Delta$-8-Cholestenol levels decreased to a minimum after $3 \mathrm{~d}$ of drug treatment (data not shown). Cholesterol was decreased by $29 \%$ from $6.6 \pm 0.2 \mu \mathrm{g} /$ well in vehicle-treated cultures to $4.7 \pm 0.1 \mu \mathrm{g} /$ well in cultures treated for $3 \mathrm{~d}$ with $30 \mathrm{nM}$ rosuvastatin $(p<0.05)$. In the same cultures, $\Delta$-8-cholestenol was decreased by $72 \%$ from $119 \pm 17$ to $33 \pm 8 \mathrm{ng} /$ well $(p<$ $0.01) . \Delta$-8-Cholestenol is an intermediate sterol in the biosynthesis of cholesterol, so a decrease in the ratio of $\Delta-8$-cholestenol to cholesterol indicates that rosuvastatin inhibited sterol biosynthesis in cortical cultures. Rosuvastatin reduced the ratio of cortical culture $\Delta$-8-cholestenol to cholesterol by more than threefold after $3 \mathrm{~d}$. As shown in Figure 3, rosuvastatin decreased the ratio of $\Delta$-8-cholestenol to cholesterol in a concentration-dependent manner with an $\mathrm{IC}_{50}$ value of $5 \mathrm{nM}$ (95\% confidence interval, 4-10 nм).

\section{Reversal of neuroprotection by mevalonate or cholesterol}

The product of HMG-CoA reductase, mevalonate, is a biosynthetic intermediate in several biochemical pathways, including cholesterol biosynthesis. To determine whether depletion of mevalonate and subsequent depletion of cholesterol was involved in the prevention of NMDA excitotoxicity, cultures were cotreated with simvastatin alone or with simvastatin in combination with

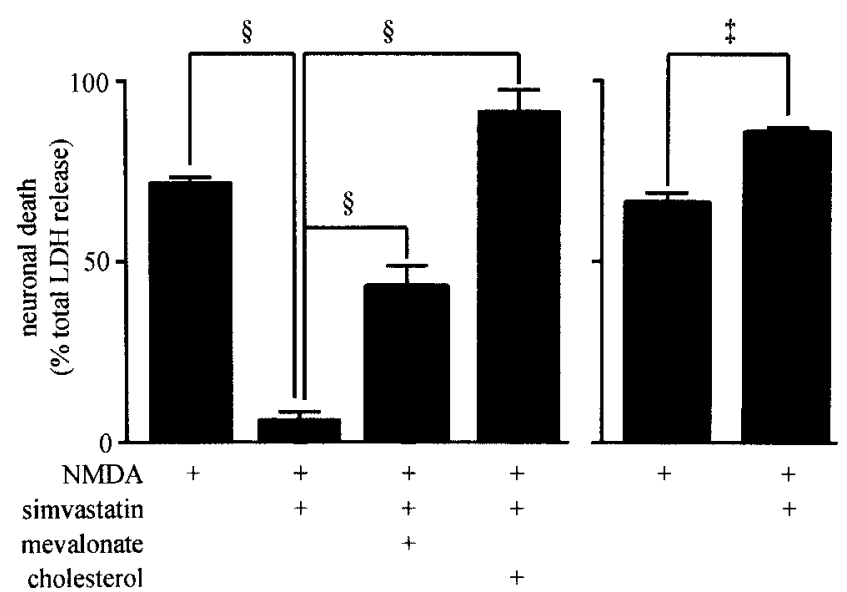

Figure 4. Resistance to excitotoxicity was prevented by coincubation with either mevalonate or cholesterol. In the experiment shown on the left side of the bar graph, before NMDA exposure, cortical cultures were left untreated, treated for $6 \mathrm{~d}$ with $100 \mathrm{~nm}$ simvastatin alone, or treated with both simvastatin and either $1 \mathrm{~mm}$ mevalonate or $50 \mu \mathrm{m}$ cholesterol. NMDAinduced LDH release after cotreatment with simvastatin and either mevalonate or cholesterol was increased compared with NMDA-induced LDH release after treatment with simvastatin alone. In the experiment shown on the right side of the bar graph, cultures were treated with vehicle or cholesterol for $6 \mathrm{~d}$ before NMDA exposure. Cholesterol treatment increased NMDAinduced LDH release. $\S, p<0.001 ; \ddagger, p<0.01$.

either $1 \mathrm{~mm}$ mevalonate or $50 \mu \mathrm{M}$ cholesterol. As shown in Figure 4 (left), cotreatment with either mevalonate or cholesterol prevented the neuroprotective action of simvastatin. In simvastatintreated cultures, NMDA-induced LDH release was reduced from $71 \pm 2$ to $6 \pm 2 \%$ of total $\mathrm{LDH}(p<0.001)$. In cultures exposed to simvastatin and either mevalonate or cholesterol, the extent of NMDA-induced LDH release was $43 \pm 5$ and $91 \pm 7 \%$ of total LDH release, respectively, significantly more than that observed in cultures treated with simvastatin alone $(p<0.001)$. Cotreatment with cholesterol not only prevented the protective effect of simvastatin but increased NMDA-induced LDH release above that observed in control cultures $(91 \pm 7 \%$ compared with $71 \pm$ $2 \%$ of total LDH; $p<0.05)$. These data suggested that manipulation of cell cholesterol levels could directly alter NMDA toxicity independent of HMG-CoA reductase inhibition.

This possibility was explored in two subsequent experiments. First, the effect of chronic cholesterol loading on NMDAinduced excitotoxicity was measured. As shown in Figure 4 (right), pretreatment of cultures for $6 \mathrm{~d}$ with $50 \mu \mathrm{M}$ cholesterol increased NMDA-induced LDH release to $82 \pm 4 \%$ of total LDH release, a significant increase over that observed in vehicletreated cultures $(67 \pm 3 \%$; $p<0.01)$. This experiment suggests that loading cultures with cholesterol increases the excitotoxic effect of NMDA.

The cyclic glucose oligomer methyl- $\beta$-cyclodextrin $(\beta C D)$ has been used to selectively deplete cell membranes of cholesterol, while leaving membrane phospholipid content intact (Kilsdonk et al., 1995; Klein et al., 1995; Simons et al., 1998; Martens et al., 2000). $\beta C D$ was used to investigate the effect of acute cholesterol depletion and repletion on the excitotoxicity of NMDA in cortical cultures. As shown in Figure 5, NMDA-induced LDH release was reduced from $77 \pm 2.2 \%$ in control cultures to $13 \pm$ $0.2 \%$ of total $\mathrm{LDH}(p<0.001)$ in cultures treated for $20 \mathrm{~min}$ with $1 \% \beta \mathrm{CD}$, followed by incubation in HCSS for $15 \mathrm{~min}$ before NMDA exposure. Treatment of cultures for 20 min with $1 \% \beta C D$ to deplete cholesterol, followed by a $15 \mathrm{~min}$ treatment with $2 \mathrm{~mm}$ 


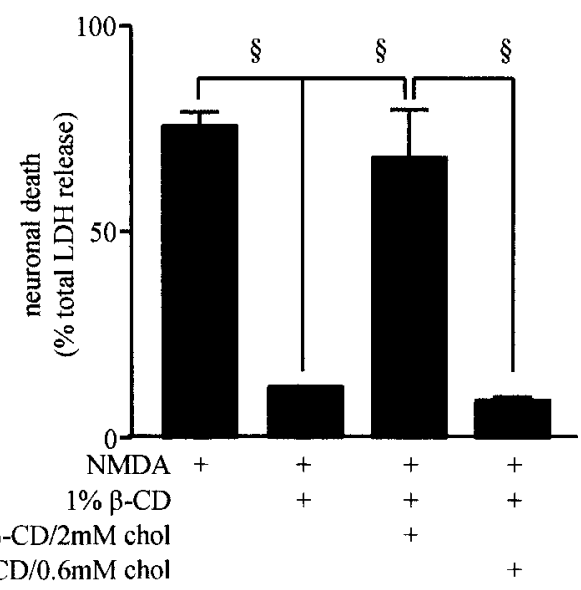

Figure 5. Specific depletion of membrane cholesterol with $\beta C D$ prevents NMDA-induced excitotoxicity. Before NMDA exposure, cultures were left untreated or treated with $1 \% \beta C D$ for $20 \mathrm{~min}$. $\beta C D$-treated cultures were then left untreated or treated for $15 \mathrm{~min}$ with the indicated dilutions of $\beta C D /$ cholesterol. Cholesterol depletion prevented and cholesterol repletion restored NMDA excitotoxicity. $\S, p<0.001$.

cholesterol complexed with $2.5 \% \beta \mathrm{CD}$ to replace cholesterol, restored NMDA-induced $\mathrm{LDH}$ release back to control levels $(64 \pm 8 \%$ of total LDH). This effect of cholesterol was concentration dependent, because restoring cholesterol levels by treatment with $0.6 \mathrm{~mm}$ cholesterol complexed with $0.7 \% \beta C D$ did not prevent the protective effect of cholesterol depletion by $1 \% \beta C D$ treatment. These data indicate that treatments that acutely deplete cell cholesterol produced a neuroprotective effect similar to that observed after chronic statin treatment.

\section{NMDAR function}

Whole-cell patch-clamp electrophysiological recordings and calcium microfluorimetry were used to investigate effects on NMDAR function caused by chronic exposure of mouse cortical cultures to simvastatin. As shown in Figure 6, treatment of cortical cultures for 4-6 d with simvastatin decreased NMDAinduced whole-cell currents and current density (data not shown) by $25 \%$ (mean \pm SEM; untreated: $1947 \pm 124$ pA, 36 $\mathrm{pA} / \mathrm{pF}, n=33$; simvastatin: $1479 \pm 119 \mathrm{pA}, 27 \mathrm{pA} / \mathrm{pF}, n=22 ; p<$ 0.01 for both whole-cell current and current density). In contrast, the magnitude of the $300 \mu \mathrm{M}$ NMDA-induced increase in intraneuronal calcium concentration was not different in simvastatintreated and control cultures $\left(F_{340} / F_{380}\right.$; control: $0.330 \pm 0.008$, $n=22$ coverslips, 331 cells; simvastatin: $0.319 \pm 0.006, n=21$ coverslips, 371 cells). These data indicate that, although statin treatment slightly reduces NMDA-induced whole-cell current, this change is not reflected in reduced intraneuronal calcium concentrations.

\section{Discussion}

Here, HMG-CoA reductase inhibitors, or statins, are shown for the first time to elicit resistance of cultured neurons to NMDAinduced excitotoxic death, a potential neuroprotective action of statins distinct from their beneficial effects on cerebrovascular function.

The HMG-CoA reductase enzyme is rate limiting in cholesterol biosynthesis. The neuroprotective effect described here is attributable to inhibition of cholesterol biosynthesis and depletion of cholesterol or a metabolic product of cholesterol. The rank order of neuroprotective potency of statins is generally consistent with the rank order of potency reported for inhibition of
A

\begin{tabular}{|l|c|c|}
\hline Treatment & $\mathrm{I}_{\mathrm{NMDA}}(\mathrm{pA})$ & $\Delta \mathrm{R}\left(\mathrm{F}_{340} / \mathrm{F}_{380}\right)$ \\
\hline control & -1947 & 0.330 \\
simva & $-1479 \ddagger$ & 0.319 \\
\hline
\end{tabular}
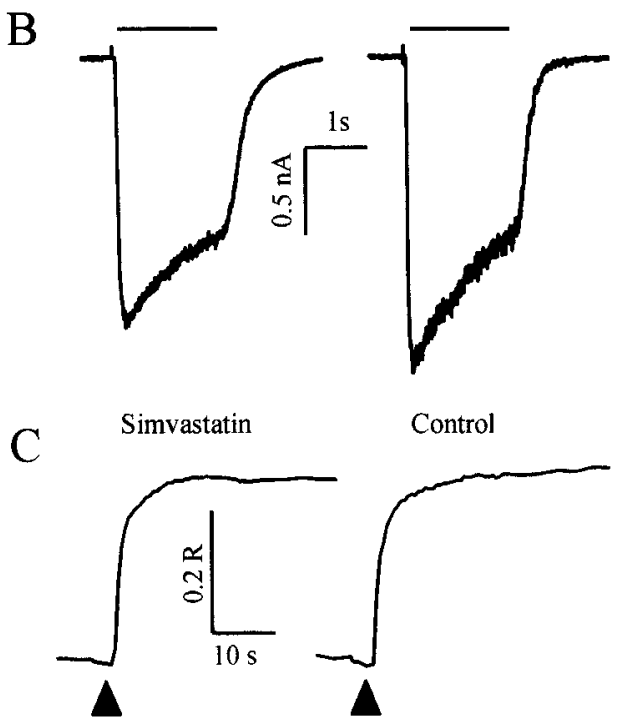

Figure 6. Simvastatin reduces NMDA-induced whole-cell currents but does not affect the NMDA-induced increase in intraneuronal calcium concentration. $A$, The table provides mean and SEM peak NMDA-induced whole-cell current ( $n=22$ cells for simvastatin; $n=33$ cells for control; $\ddagger, p<0.01$ ) and fura- $2 \Delta R$ (simvastatin, $n=331$ cells from 22 coverslips; control, $n=$ 371 cells from 21 coverslips) obtained in simvastatin-treated and control cultures. Mean data were obtained from experiments conducted in three cultures. $B$, Representative traces of NMDA-induced whole-cell currents. The horizontal bar indicates the application of $100 \mu \mathrm{m}$ NMDA. C, Representative traces of NMDA-induced changes in the fura- 2 fluorescence intensity ratio. The black triangles indicate the initiation of application of $300 \mu \mathrm{m}$ NMDA.

either the purified HMG-CoA reductase enzyme or of cellular cholesterol biosynthesis. For example, the rank order potencies and $\mathrm{IC}_{50}$ values (as nanomolar concentrations) for inhibition of the purified catalytic domain of human HMG-CoA reductase were rosuvastatin $(5.4)=$ atorvastatin $(8.2)>$ simvastatin (11.2) > pravastatin (44.1) and for inhibition of cultured rat hepatocyte cholesterol synthesis were rosuvastatin $(0.16)>$ atorvastatin $(1.16)=$ simvastatin $(2.74)=$ pravastatin (6.93) (McTaggart et al., 2001). The presence of specific uptake mechanisms increases the potency of rosuvastatin and pravastatin, but not simvastatin, by $>100$-fold in rat hepatocytes compared with fibroblasts. It is not known whether such uptake mechanisms are present in mouse cortical neurons.

$\Delta-8$-Cholestenol is an intermediate in the biosynthesis of cholesterol and has a shorter half-life than cholesterol. Rosuvastatin decreased both cholesterol and $\Delta$-8-cholestenol levels, and decreased the ratio of $\Delta-8$-cholestenol to cholesterol in cortical cultures. These changes are consistent with depletion of cortical culture sterols after inhibition of HMG-CoA reductase. The observed decrease in the ratio of $\Delta$-8-cholestenol to cholesterol indicates that rosuvastatin inhibited de novo cholesterol biosynthesis in cortical cultures. Treatment with rosuvastatin decreased culture cholesterol by inhibiting cholesterol biosynthesis. The time course and potency with which rosuvastatin reduced cell sterols closely matched the time course of mevastatin and simva- 
statin and the potency of rosuvastatin to protect cortical neurons from excitoxicity. Future studies should determine whether the rank order potency for inhibition of cholesterol biosynthesis matches the rank order potency for neuroprotection.

Statins inhibit cholesterol biosynthesis more potently than other mevalonate-dependent biochemical pathways. Isoprenylation of proteins, which is dependent on mevalonate biosynthesis, is inhibited by lovastatin at micromolar concentrations, 1000fold higher than the concentration required for inhibition of cholesterol biosynthesis in the same cells (Sinensky et al., 1990). Furthermore, in cortical cultures, micromolar concentrations of statins are highly toxic (Tanaka et al., 2000), an effect that is prevented by cotreatment with the isoprenoid precursor geranylgeranyl pyrophosphate. Similar observations were made in the cultures used in this study, and these observations suggest that the neuroprotective effect described here is not attributable to inhibition of isoprenoid biosynthesis. The neuroprotective potency of statins observed in this study is consistent with inhibition of cholesterol biosynthesis and less consistent with inhibition of other mevalonate-dependent biochemical pathways.

Resistance to excitotoxicity was substantially attenuated by coincubation with either mevalonate or cholesterol. Cotreatment with mevalonate restores cholesterol biosynthesis as well as other mevalonate-dependent biosynthetic pathways. The ability of cholesterol to reverse statin neuroprotection implies that inhibition of cholesterol synthesis and subsequent decrease in cell cholesterol content is essential to the statin neuroprotective mechanism (Michikawa and Yanagisawa, 1999).

Finally, two experiments suggest that manipulation of cell cholesterol without statins produced changes in the susceptibility of the cultures to NMDA-induced excitotoxicity. Treatment with cholesterol increased susceptibility to NMDA excitotoxicity. In addition, $\beta C D$, which extracts membrane cholesterol (Kilsdonk et al., 1995; Klein et al., 1995), reduced susceptibility to NMDAinduced excitotoxicity, an effect reversed by subsequent treatment with a cholesterol/ $\beta C D$ mixture, which restores extracted cell cholesterol (Klein et al., 1995). Taken together, these results indicate that decreased cholesterol content caused by inhibition of HMG-CoA reductase protects cultured cortical neurons from NMDA-induced excitotoxicity.

Two previous studies suggest that exposure of cultured neurons to statins triggers neuronal apoptosis (Michikawa and Yanagisawa, 1999; Tanaka et al., 2000). Tanaka et al. (2000) reported that simvastatin caused neurotoxicity with an $\mathrm{EC}_{50}$ value of $3 \mu \mathrm{M}$ because of depletion of the isoprenoid ggPP and not because of depletion of intermediates in cholesterol biosynthesis. In cultures used in the present study, concentrations of simvastatin or mevastatin $>3 \mu \mathrm{M}$ also caused the death of cultured neurons, which was reversed by ggPP but not by fPP (data not shown), but the protection against NMDA excitotoxicity reported here was apparent at concentrations $>300$-fold less than those that cause toxicity. The potency of the neuroprotective effect of statins because of inhibition of cholesterol biosynthesis differentiates this effect from the cytotoxicity of statins because of inhibition of ggPP biosynthesis.

Michikawa and Yanagisawa (1999) also observed apoptosis of statin-treated cultured neurons and concluded that the toxicity of statins was caused by inhibition of cholesterol synthesis. These investigators used neuronal cultures $6 \mathrm{hr}$ after plating and suggested that survival of newly plated neurons may strictly require cholesterol biosynthesis to support formation of new membranes.

The effect of reduced cortical culture cholesterol on NMDAR- mediated excitotoxic events is unknown. The modest decrease in NMDAR current observed in simvastatin-treated neurons may account partly for the observed neuroprotective efficacy of simvastatin, however the identical treatment decreased neuronal death by $>90 \%$. Under conditions used here, NMDAR-mediated excitotoxicity is largely calcium dependent, and the peak NMDAinduced intraneuronal calcium concentration in these cultures was unchanged by statin treatment. The cause of this discrepancy is unknown, but it could be attributable to either to insufficient sensitivity of the calcium measurements to detect decreased intraneuronal calcium, or to altered permeability of the cationic nonselective NMDAR pore such that the whole-cell current was reduced without reducing the resulting intraneuronal calcium load. Finally, NMDAR-1 immunocytochemistry revealed similar levels of NMDAR expression in statin-protected and untreated cultures. These data suggest that statins reduce susceptibility of cortical neurons to NMDA excitotoxicity by acting downstream of NMDAR function.

Depletion of cholesterol from plasma membrane microdomains may modulate key steps in the excitotoxic cascade downstream of NMDAR function. NMDAR-mediated calcium influx activates NOS (nNOS). nNOS-mediated production of nitric oxide and subsequent formation of reactive oxygen radicals is a cause of excitotoxic neuronal death, and inhibitors of nNOS protect cultured cortical neurons from excitotoxicity (Dawson et al., 1993). nNOS forms a tri-partite complex with the NMDAR and the postsynaptic density protein PSD-95 (Christopherson et al., 1999). nNOS and PSD-95 are found in sterol-rich plasma-membrane microdomains called lipid rafts (Perez and Bredt, 1998; Topinka and Bredt, 1998; Keshet et al., 1999), and depletion of membrane cholesterol alters localization, composition, and function of protein complexes associated with rafts (Martens et al., 2000; Ehehalt et al., 2003). Statins may alter the sterol content of lipid rafts, thereby uncoupling nNOS activation from NMDAR-mediated calcium flux. Antisense suppression of PSD-95 selectively uncouples NMDAR-induced calcium flux from nNOS activation and protects cultured neurons from NMDA excitotoxicity (Sattler et al., 1999), and peptides that dissociate NMDAR and PSD-95 prevent excitotoxic neuronal death and reduce infarct volume after cerebral ischemia (Aarts et al., 2002). Future studies should determine whether cholesterol depletion is neuroprotective because of changes in nNOS activation or NMDAR composition resulting from altered lipid raft sterol composition.

Laufs et al. (2000) have reported that 2 week pretreatment of mice with simvastatin, lovastatin, atorvastatin, or mevastatin reduces infarct size and neurological deficits caused by subsequent middle cerebral artery occlusion in mice (Endres et al., 1998; Amin-Hanjani et al., 2001). Recently, these investigators have described a more potent neuroprotective effect of rosuvastatin (Laufs et al., 2002). The proposed cerebrovascular neuroprotective mechanism of statins differs from the neuroprotective mechanism described here, in that cerebrovascular effects occurred without a change in plasma cholesterol and seemed to require reduced endothelial cell isoprenoids, not reduced cholesterol (Laufs and Liao, 1998; Laufs et al., 1998). Furthermore, whereas simvastatin can reduce brain cholesterol biosynthesis in vivo (Fassbender et al., 2001, 2002; Simons et al., 2002), this likely occurs at doses far exceeding those used by Endres et al. (1998) and could not occur independent of reduced plasma cholesterol. Additional experiments are needed to determine whether the neuroprotective effect described here occurs in vivo and whether 
cerebrovascular and central neuroprotective effects could be synergistic.

Clinically, it would be reasonable to postulate that vascular effects of statins might predominantly influence stroke incidence, whereas the neuroprotective mechanism described here might predominantly affect the severity of resultant neurological damage. Reliably distinguishing these, and which mechanism is responsible for which effect, is beyond the scope of data from currently available, large-scale clinical studies. Reduced incidence of stroke has been observed in middle-aged men treated with simvastatin (Scandinavian Simvastatin Survival Study Group, 1994) or pravastatin [The Long-Term Intervention with Pravastatin in Ischemic Disease (LIPID) Study Group, 1998], although pravastatin does not readily cross the blood-brain barrier. However, pravastatin did not reduce stroke incidence in elderly patients (Shepherd et al., 2002); this might be because of more advanced vascular disease in the elderly being less amenable to cerebrovascular protection. Detailed assessment of the extent and severity of neurological damage was not performed in these studies, making it difficult to judge whether the neuroprotection demonstrated here could have afforded any clinical benefit.

In conclusion, increased resistance of statin-treated cultured neurons to NMDAR-mediated excitotoxic death is attributable to reduced cell cholesterol, occurs in the absence of vascular elements, and represents an additional, plausible, "direct" neuroprotective mechanism.

\section{References}

Aarts M, Liu Y, Liu L, Besshoh S, Arundine M, Gurd JW, Wang YT, Salter MW, Tymianski M (2002) Treatment of ischemic brain damage by perturbing NMDA receptor-PSD-95 protein interactions. Science 298:846-850

Amin-Hanjani S, Stagliano NE, Yamada M, Huang PL, Liao JK, Moskowitz MA (2001) Mevastatin, an HMG-CoA reductase inhibitor, reduces stroke damage and upregulates endothelial nitric oxide synthase in mice. Stroke 32:980-986.

Christopherson KS, Hillier BJ, Lim WA, Bredt DS (1999) PSD-95 assembles a ternary complex with the $\mathrm{N}$-methyl-D-aspartic acid receptor and a bivalent neuronal NO synthase PDZ domain. J Biol Chem 274:27467-27473.

Dawson VL, Dawson TM, Bartley DA, Uhl GR, Snyder SH (1993) Mechanisms of nitric oxide-mediated neurotoxicity in primary brain cultures. J Neurosci 13:2651-2661.

Ehehalt R, Keller P, Haass C, Thiele C, Simons K (2003) Amyloidogenic processing of the Alzheimer beta-amyloid precursor protein depends on lipid rafts. J Cell Biol 160:113-123.

Endres M, Laufs U, Huang Z, Nakamura T, Huang P, Moskowitz MA, Liao JK (1998) Stroke protection by 3-hydroxy-3-methylglutaryl (HMG)-CoA reductase inhibitors mediated by endothelial nitric oxide synthase. Proc Natl Acad Sci USA 95:8880-8885.

Fassbender K, Simons M, Bergmann C, Stroick M, Lutjohann D, Keller P, Runz H, Kuhl S, Bertsch T, von Bergmann K, Hennerici M, Beyreuther K, Hartmann T (2001) Simvastatin strongly reduces levels of Alzheimer's disease beta-amyloid peptides Abeta 42 and Abeta 40 in vitro and in vivo. Proc Natl Acad Sci USA 98:5856-5861.

Fassbender K, Stroick M, Bertsch T, Ragoschke A, Kuehl S, Walter S, Walter J, Brechtel K, Muehlhauser F, von Bergmann K, Lutjohann D (2002) Effects of statins on human cerebral cholesterol metabolism and secretion of Alzheimer amyloid peptide. Neurology 59:1257-1258.

Heart Protection Study Collaborative Group (2002) MRC/BHF Heart Protection Study of cholesterol lowering with simvastatin in 20,536 high-risk individuals: a randomised placebo-controlled trial. Lancet 360:7-22.

Jick H, Zornberg GL, Jick SS, Seshadri S, Drachman DA (2000) Statins and the risk of dementia. Lancet [Erratum (2001) 357:562] 356:1627-1631.

Keshet GI, Ovadia H, Taraboulos A, Gabizon R (1999) Scrapie-infected mice and PrP knockout mice share abnormal localization and activity of neuronal nitric oxide synthase. J Neurochem 72:1224-1231.
Kilsdonk EP, Yancey PG, Stoudt GW, Bangerter FW, Johnson WJ, Phillips MC, Rothblat GH (1995) Cellular cholesterol efflux mediated by cyclodextrins. J Biol Chem 270:17250-17256.

Klein U, Gimpl G, Fahrenholz F (1995) Alteration of the myometrial plasma membrane cholesterol content with beta-cyclodextrin modulates the binding affinity of the oxytocin receptor. Biochemistry 34:13784-13793.

Laufs U, Liao JK (1998) Post-transcriptional regulation of endothelial nitric oxide synthase mRNA stability by Rho GTPase. J Biol Chem 273:24266-24271.

Laufs U, La FV, Plutzky J, Liao JK (1998) Upregulation of endothelial nitric oxide synthase by HMG CoA reductase inhibitors. Circulation 97:1129-1135.

Laufs U, Gertz K, Huang P, Nickenig G, Bohm M, Dirnagl U, Endres M (2000) Atorvastatin upregulates type III nitric oxide synthase in thrombocytes, decreases platelet activation, and protects from cerebral ischemia in normocholesterolemic mice. Stroke 31:2442-2449.

Laufs U, Wassmann S, Hilgers S, Ribaudo N, Bohm M, Nickenig G (2001) Rapid effects on vascular function after initiation and withdrawal of atorvastatin in healthy, normocholesterolemic men. Am J Cardiol 88:1306-1307.

Laufs U, Gertz K, Dirnagl U, Bohm M, Nickenig G, Endres M (2002) Rosuvastatin, a new HMG-CoA reductase inhibitor, upregulates endothelial nitric oxide synthase and protects from ischemic stroke in mice. Brain Res 942:23-30.

Martens JR, Navarro-Polanco R, Coppock EA, Nishiyama A, Parshley L, Grobaski TD, Tamkun MM (2000) Differential targeting of Shaker-like potassium channels to lipid rafts. J Biol Chem 275:7443-7446.

McTaggart F, Buckett L, Davidson R, Holdgate G, McCormick A, Schneck D, Smith G, Warwick M (2001) Preclinical and clinical pharmacology of rosuvastatin, a new 3-hydroxy-3-methylglutaryl coenzyme A reductase inhibitor. Am J Cardiol 87:28B-32B.

Michikawa M, Yanagisawa K (1999) Inhibition of cholesterol production but not of nonsterol isoprenoid products induces neuronal cell death. J Neurochem 72:2278-2285.

Perez AS, Bredt DS (1998) The N-terminal PDZ-containing region of postsynaptic density-95 mediates association with caveolar-like lipid domains. Neurosci Lett 258:121-123.

Sacks FM, Pfeffer MA, Moye LA, Rouleau JL, Rutherford JD, Cole TG, Brown L, Warnica JW, Arnold JM, Wun CC, Davis BR, Braunwald E (1996) The effect of pravastatin on coronary events after myocardial infarction in patients with average cholesterol levels. Cholesterol and Recurrent Events Trial investigators. N Engl J Med 335:1001-1009.

Sattler R, Tymianski M (2001) Molecular mechanisms of glutamate receptor-mediated excitotoxic neuronal cell death. Mol Neurobiol 24:107-129.

Sattler R, Xiong Z, Lu WY, Hafner M, MacDonald JF, Tymianski M (1999) Specific coupling of NMDA receptor activation to nitric oxide neurotoxicity by PSD-95 protein. Science 284:1845-1848.

Scandinavian Simvastatin Survival Study Group (1994) Randomised trial of cholesterol lowering in 4444 patients with coronary heart disease: the Scandinavian Simvastatin Survival Study (4S). Lancet 344:1383-1389.

Shepherd J, Cobbe SM, Ford I, Isles CG, Lorimer AR, MacFarlane PW, McKillop JH, Packard CJ (1995) Prevention of coronary heart disease with pravastatin in men with hypercholesterolemia. West of Scotland Coronary Prevention Study Group. N Engl J Med 333:1301-1307.

Shepherd J, Blauw GJ, Murphy MB, Bollen EL, Buckley BM, Cobbe SM, Ford I, Gaw A, Hyland M, Jukema JW, Kamper AM, MacFarlane PW, Meinders AE, Norrie J, Packard CJ, Perry IJ, Stott DJ, Sweeney BJ, Twomey C, Westendorp RG, PROSPER study group. Prospective Study of Pravastatin in the Elderly at Risk (2002) Pravastatin in elderly individuals at risk of vascular disease (PROSPER): a randomised controlled trial. Lancet 360:1623-1630.

Simons M, Keller P, De Strooper B, Beyreuther K, Dotti CG, Simons K (1998) Cholesterol depletion inhibits the generation of beta-amyloid in hippocampal neurons. Proc Natl Acad Sci USA 95:6460-6464.

Simons M, Schwarzler F, Lutjohann D, von Bergmann K, Beyreuther K, Dichgans J, Wormstall H, Hartmann T, Schulz JB (2002) Treatment with simvastatin in normocholesterolemic patients with Alzheimer's disease: a 26-week randomized, placebo-controlled, double-blind trial. Ann Neurol 52:346-350. 
Sinensky M, Beck LA, Leonard S, Evans R (1990) Differential inhibitory effects of lovastatin on protein isoprenylation and sterol synthesis. J Biol Chem 265:19937-19941.

Tanaka T, Tatsuno I, Uchida D, Moroo I, Morio H, Nakamura S, Noguchi Y, Yasuda T, Kitagawa M, Saito Y, Hirai A (2000) Geranylgeranylpyrophosphate, an isoprenoid of mevalonate cascade, is a critical compound for rat primary cultured cortical neurons to protect the cell death induced by 3-hydroxy-3-methylglutaryl-CoA reductase inhibition. J Neurosci 20:2852-2859.

The Long-Term Intervention with Pravastatin in Ischaemic Disease (LIPID) Study Group (1998) Prevention of cardiovascular events and death with pravastatin in patients with coronary heart disease and a broad range of initial cholesterol levels. N Engl J Med 339:1349-1357.

Topinka JR, Bredt DS (1998) N-terminal palmitoylation of PSD-95 regulates association with cell membranes and interaction with $\mathrm{K}+$ channel Kv1.4. Neuron 20:125-134.

Warshafsky S, Packard D, Marks SJ, Sachdeva N, Terashita DM, Kaufman G, Sang K, Deluca AJ, Peterson SJ, Frishman WH (1999) Efficacy of 3-hydroxy-3-methylglutaryl coenzyme A reductase inhibitors for prevention of stroke. J Gen Intern Med 14:763-774.

Wolozin B, Kellman W, Ruosseau P, Celesia GG, Siegel G (2000) Decreased prevalence of Alzheimer disease associated with 3-hydroxy-3-methyglutaryl coenzyme A reductase inhibitors. Arch Neurol 57:1439-1443. 